International Mathematical Forum, Vol. 8, 2013, no. 31, 1515 - 1522

HIKARI Ltd, www.m-hikari.com

http://dx.doi.org/10.12988/imf.2013.37131

\title{
Weakly Convex and Weakly Connected Independent Dominations in the Corona of Graphs
}

\author{
Rene E. Leonida \\ Mathematics Department \\ College of Natural Sciences and Mathematics \\ Mindanao State University \\ General Santos City, Philippines \\ leonida.rene@yahoo.com \\ Sergio R. Canoy, Jr. \\ Mathematics Department \\ College of Science and Mathematics \\ MSU-Iligan Institute of Technology \\ Iligan City, Philippines \\ serge_canoy@yahoo.com
}

Copyright (C) 2013 Rene E. Leonida and Sergio R. Canoy, Jr. This is an open access article distributed under the Creative Commons Attribution License, which permits unrestricted use, distribution, and reproduction in any medium, provided the original work is properly cited.

\begin{abstract}
In this paper we constructed a connected graph with a preassigned order, weakly convex domination number, convex domination number, weakly connected independent domination number, and upper weakly connected independent domination number; characterized the weakly convex set, the weakly convex dominating set, and the weakly connected independent dominating set of the corona graph. As direct consequence, the weakly convexity number, weakly convex domination number, and the weakly connected independent domination number of this graph were obtained.
\end{abstract}

Mathematics Subject Classification: $05 \mathrm{C} 12$ 
Keywords: domination, weakly convex, weakly connected, independent domination

\section{Introduction and Preliminary Results}

Let $G=(V(G), E(G))$ be a connected graph. For any vertex $v \in V(G)$, the open neighborhood of $v$ is the set $N(v)=\{u \in V(G): u v \in E(G)\}$ and the closed neighborhood of $v$ is the set $N[v]=N(v) \cup\{v\}$. For a set $X \subseteq V(G)$, the open neighborhood of $X$ is $N(X)=\bigcup_{v \in X} N(v)$ and the closed neighborhood of $X$ is $N[X]=X \cup N(X)$. For any two vertices $u$ and $v$ of $G$, the distance $d_{G}(u, v)$ is the length of the shortest $u-v$ path in $G$. A $u-v$ path of length $d_{G}(u, v)$ is called $u-v$ geodesic. A set $C \subseteq V(G)$ is called weakly convex in $G$ if for every two vertices $u, v \in C$, there exists a $u$-v geodesic whose vertices belong to $C$, or equivalently, if for every two vertices $u, v \in C, d_{\langle C\rangle}(u, v)=d_{G}(u, v)$, where $\langle C\rangle$ is the graph induced by $C$. A set $C \subseteq V(G)$ is called convex in $G$ if for every two vertices $u, v \in C$, the vertex-set of every $u-v$ geodesic is contained in $C$. The weak convexity number of $G$, denoted by wcon $(G)$, is the cardinality of a maximum weakly convex proper subset of $V(G)$. A subset $S$ of $V(G)$ is an independent set if for every $x, y \in S, x y \notin E(G)$. A subset $S$ of $V(G)$ is called weakly connected if the subgraph $\langle S\rangle_{w}=\left(N_{G}[S], E_{W}\right)$ weakly induced by $S$, is connected, where $E_{W}$ is the set of all edges with at least one vertex in $S$.

A subset $S$ of $V(G)$ is a dominating set of $G$ if for every $v \in V(G) \backslash S$, there exists $u \in S$ such that $u v \in E(G)$. The domination number $\gamma(G)$ of $G$ is the smallest cardinality of a dominating set of $G$. A dominating set of $G$ which is also weakly convex (respectively, convex) is called a weakly convex (respectively, convex) dominating set of $G$. The weakly convex (respectively, convex) domination number $\gamma_{\text {wcon }}(G)$ (respectively, $\gamma_{c o n}(G)$ ) of $G$ is the smallest cardinality of a weakly convex (respectively, convex) dominating set of $G$. Lemanska [5] discussed the relationship of the convex and weakly convex domination numbers, including their relationships with other domination parameters. In [4], Janakiraman and Alphonse found bounds for the weakly convex domination number of a graph and its complement in terms of degree, diameter and other graph parameters, and characterized graphs for which bounds are attained.

A dominating set of $G$ which is also independent is called is an independent dominating set of $G$. The independent domination number $i(G)$ of $G$ is the minimum cardinality of an independent dominating set of $G$. An independent dominating set of $G$ which is weakly connected is called a weakly connected independent dominating set of $G$. The weakly connected independent domination number $i_{w}(G)$ of $G$ is the smallest cardinality of a weakly connected independent dominating set of $G$. Similarly, the upper weakly connected 
independent domination number $\beta_{w}(G)$ is the largest cardinality of a weakly connected independent dominating set of $G$. In [1], Dunbar, et al., showed that every connected graph has a weakly connected independent dominating set. Thus, for a connected graph $G$, the weakly connected independent domination number $i_{w}(G)$ and the upper weakly connected independent domination number $\beta_{w}(G)$ always exist. Relations of these parameters with other domination parameters are also given.

Let $G$ and $H$ be graphs of order $m$ and $n$, respectively. The corona $G \circ H$ of $G$ and $H$ is the graph obtained by taking one copy of $G$ and $m$ copies of $H$, and then joining the $i t h$ vertex of $G$ to every vertex of the $i t h$ copy of $H$. For every $v \in V(G)$, denote by $H^{v}$ the copy of $\mathrm{H}$ whose vertices are attached one by one to the vertex $v$. Denote by $v+H^{v}$ the subgraph of the corona $G \circ H$ corresponding to the join $\langle\{v\}\rangle+H^{v}$.

In this paper, we assume that $G=(V(G), E(G))$ is a simple undirected graph. A path with vertices $v_{1}, v_{2}, \ldots, v_{n}$, with endpoints $v_{1}$ and $v_{n}$ is denoted by $P_{n}=P\left(v_{1}, v_{n}\right)=\left[v_{1}, v_{2}, \ldots, v_{n}\right]$.

The following results can be easily verified.

Proposition 1.1 Let $n$ be a positive integer. Then

$$
\gamma_{\text {wcon }}\left(P_{n}\right)=\gamma_{\text {con }}\left(P_{n}\right)= \begin{cases}1, & \text { if } n \leq 3 \\ n-2, & \text { if } n \geq 4\end{cases}
$$

Proposition 1.2 Let $n \geq 2$ be a positive integer. Then $i_{w}\left(P_{n}\right)=\left\lfloor\frac{n}{2}\right\rfloor$ and $\beta_{w}\left(P_{n}\right)=\left\lceil\frac{n}{2}\right\rceil$.

We construct a graph with a preassigned order and some domination parameters.

Theorem 1.3 (Realization Problem) Given positive integers $a, b, c, d$, and $n$ with $4 \leq a<b<c<d<n$, there exists a graph $G$ with $|V(G)|=n$, $\gamma_{w c o n}(G)=a, \gamma_{c o n}(G)=b, i_{w}(G)=c$, and $\beta_{w}(G)=d$.

Proof: Let $P_{a+1}$ be the path $\left[x_{1}, x_{2}, \ldots, x_{a}, x_{a+1}\right]$ and let $H$ be the graph obtained from $P_{a+1}$ by adding the paths $\left[x_{1}, w_{k}, x_{3}\right]$ for $k=1,2, \ldots, b-a$ and new vertices $z_{1}, z_{2}, \ldots, z_{n-c-d}$, and forming the complete graph $K_{n-c-d+2}$, where $V\left(K_{n-c-d+2}\right)=\left\{x_{1}, x_{2}, z_{1}, \ldots, z_{n-c-d}\right\}$. Consider the following cases:

Case 1. Suppose $a$ is even.

Let $G$ be the graph obtained from $H$ by adding the edges $x_{a} u_{i}$ for $i=$ $1,2, \ldots, c-\frac{a}{2}-1$ and $x_{1} v_{j}$ for $j=1,2, \ldots, d-b+\frac{a}{2}$ (see Figure 2). Then $\left\{x_{1}, x_{2}, \ldots, x_{a}\right\}$ is a minimum weakly convex dominating set of $G,\left\{x_{1}, \ldots, x_{a}\right\} \cup$ $\left\{w_{1}, \ldots, w_{b-a}\right\}$ is a minimum convex dominating set of $G,\left\{x_{1}, x_{3}, \ldots, x_{a+1}\right\} \cup$ 


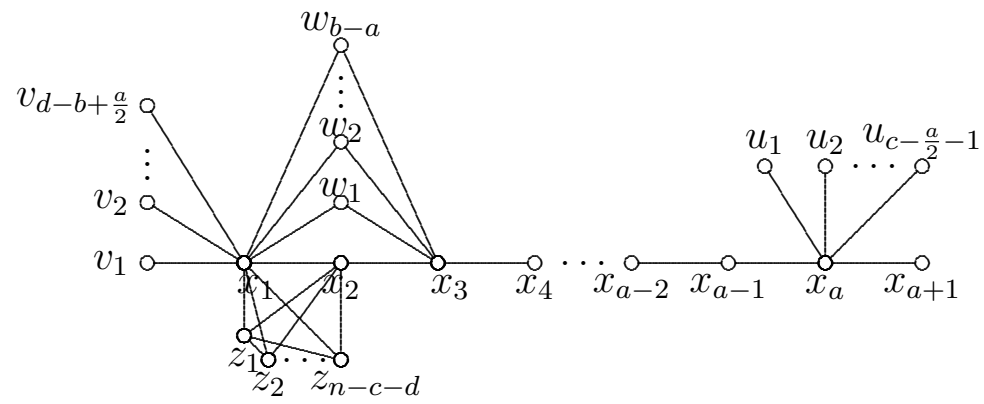

Figure 1: The graph $G$ when $a$ is even.

$\left\{u_{1}, u_{2}, \ldots, u_{c-\frac{a}{2}-1}\right\}$ is a minimum weakly connected independent dominating set of $G$, and $\left\{x_{2}, x_{4}, \ldots, x_{a}\right\} \cup\left\{w_{1}, \ldots, w_{b-a}\right\} \cup\left\{v_{1}, v_{2}, \ldots, v_{d-b+\frac{a}{2}}\right\}$ is a maximum weakly connected independent dominating set of $G$. Therefore, $\gamma_{w c o n}(G)=a$, $\gamma_{\text {con }}(G)=b, i_{w}(G)=c$, and $\beta_{w}(G)=d$. Moreover, $|V(G)|=n$.

Case 2. Suppose $a$ is odd.

Let $G$ be the graph obtained from $H$ by adding the edges $x_{a-1} u_{i}$ for $i=1,2, \ldots c-\frac{a+1}{2}$ and $x_{1} v_{j}$ for $j=1,2, \ldots, d-b+\frac{a-1}{2}$ (see Figure 3 ). Then

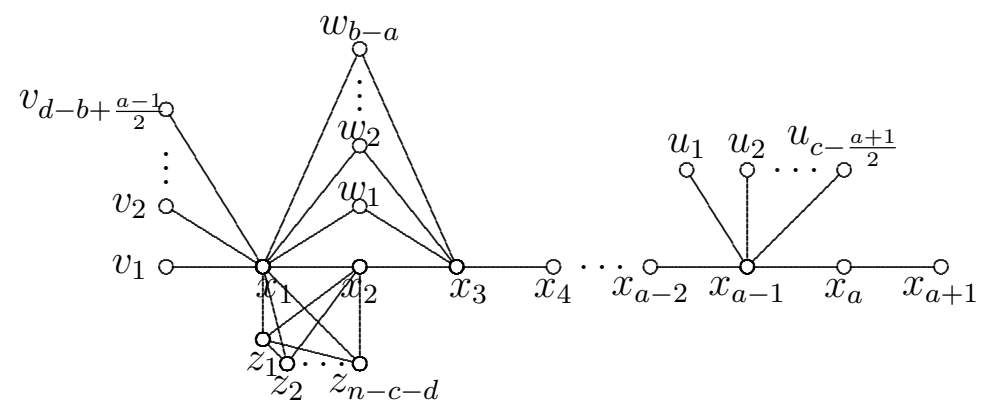

Figure 2: The graph $G$ when $a$ is odd.

$\gamma_{w c o n}(G)=a, \gamma_{c o n}(G)=b$, and $|v(G)|=n$. The set $\left\{x_{1}, x_{3}, \ldots, x_{a-1}, x_{a+1}\right\} \cup$ $\left\{u_{1}, u_{2}, \ldots, u_{c-\frac{a+1}{2}}\right\}$ is a minimum weakly connected independent dominating set of $G$ and the set $\left\{x_{2}, x_{4}, \ldots, x_{a}\right\} \cup\left\{w_{1}, \ldots, w_{b-a}\right\} \cup\left\{v_{1}, v_{2}, \ldots, v_{d-b+\frac{a-1}{2}}\right\}$ is a maximum weakly connected independent dominating set of $G$. Hence, $i_{w}(G)=$ $c$ and $\beta_{w}(G)=d$.

The next result follows from Proposition 1.1 and 1.2, and Theorem 1.3.

Corollary 1.4 The parameter pairs $i_{w}$ and $\gamma_{w c o n}, i_{w}$ and $\gamma_{c o n}, \beta_{w}$ and $\gamma_{w c o n}$, and $\beta_{w}$ and $\gamma_{c o n}$ are not comparable. 


\section{Weakly Convexity}

The next result characterizes the weakly convex sets of $G \circ H$.

Theorem 2.1 Let $G$ be a connected graph and $H$ be any graph. Then a nonempty subset $C$ of $V(G \circ H)$ is a weakly convex set of $G \circ H$ if and only if

(a) $C=S_{1} \cup S$, where $S_{1}$ is a nonempty weakly convex set of $G$ and $S \subseteq \bigcup_{v \in S_{1}} V\left(H^{v}\right)$, or

(b) $\langle C\rangle \cong\left\langle C^{*}\right\rangle$ for some $C^{*} \subseteq V(H)$ with $\operatorname{diam}_{H}\left(\left\langle C^{*}\right\rangle\right) \leq 2$.

Proof: Suppose that $C$ is a weakly convex set of $G \circ H$. Consider the following cases:

Case 1. $C \cap V(G) \neq \varnothing$.

Let $S_{1}=C \cap V(G)$. Then $S_{1}$ is a weakly convex set of $G$. Let $x \in C \backslash V(G)$ and let $v \in V(G)$ such that $x \in V\left(H^{v}\right)$. Suppose $v \notin S_{1}$. Pick $z \in S_{1}$. Then there exists an $x-z$ geodesic $P(x, z)$ with $V(P(x, z)) \subseteq C$. This, however, is impossible because $v \in V(P(x, z))$. Therefore, $v \in S_{1}$. Let $S=\{x \in C: x \in$ $V\left(H^{v}\right)$ for $\left.v \in S_{1}\right\}$. Then $S \subseteq \bigcup_{v \in S_{1}} V\left(H^{v}\right)$ and $C=S_{1} \cup S$.

Case 2. $C \cap V(G)=\varnothing$.

Suppose that there exists $v, w \in V(G), v \neq w$, such that $C \cap V\left(H^{v}\right) \neq \varnothing$ and $C \cap V\left(H^{w}\right) \neq \varnothing$. Pick $a \in C \cap V\left(H^{v}\right)$ and $b \in C \cap V\left(H^{w}\right)$. Since $C$ is weakly convex, there exists $a$ - $b$ geodesic $P(a, b)$ such that $V(P(a, b)) \subseteq C$. This implies that $v, w \in C$, which contadicts the assumption. Thus, $C \subseteq V\left(H^{v}\right)$ for some $v \in V(G)$. If $|C|=1$, then $\operatorname{diam}_{H^{v}}(\langle C\rangle) \leq 2$. Suppose $|C| \geq 2$ and let $x, y \in C$ with $x \neq y$. Since $C$ is weakly convex, there exists $x-y$ geodesic $P(x, y)$ such that $V(P(x, y)) \subseteq C$. It follows that $\operatorname{diam}_{H^{v}}(\langle C\rangle)=$ $\operatorname{diam}_{G \circ H}(\langle C\rangle) \leq 2$. Moreover, $C$ is contained in a component of $H^{v}$. Since $H^{v} \cong H$, pick $C^{*} \subseteq V(H)$ such that $\langle C\rangle \cong\left\langle C^{*}\right\rangle$. Then $\operatorname{diam}_{H}\left(\left\langle C^{*}\right\rangle\right) \leq 2$.

For the converse, suppose that $C \cap V(G)$ is a weakly convex set of $G$, where $\langle C\rangle \cong\left\langle C^{*}\right\rangle$ for some $C^{*} \subseteq V(H)$ with $\operatorname{diam}_{H}\left(\left\langle C^{*}\right\rangle\right) \leq 2$ whenever $C \cap V(G)=\varnothing$. Let $a, b \in C$. Since $G \circ H$ is connected, there exists an $a-b$ geodesic $P(a, b)$ such that $V(P(a, b)) \subseteq C$. Hence, $C$ is a weakly convex set of $G \circ H$.

As a consequence of Theorem 2.1, we have

Corollary 2.2 Let $G$ be a connected graph of order $m$ and $H$ be any graph of order $n$. Then $w \operatorname{con}(G \circ H)=m+m n-1$.

Proof: Let $x \in V\left(H^{v}\right)$ for some $v \in V(G)$ and let $S=\left[V\left(H^{v}\right) \backslash\{x\}\right] \cup$

$\left[\bigcup_{u \in V(G) \backslash\{v\}} v\left(H^{u}\right)\right]$. Then $S \subseteq \bigcup_{v \in V(G)} V\left(H^{v}\right)$ and $|S|=m n-1$. By Theorem 2.1, $C=V(G) \cup S$ is a weakly convex set of $G \circ H$. Thus, wcon $(G \circ$ $H)=|C|=|V(G)|+|S|=m+m n-1$. 


\section{Weakly Convex Domination}

The following result characterizes the weakly convex dominating sets of $G \circ H$.

Theorem 3.1 Let $G$ be a connected graph of order $m \geq 2$ and $H$ a graph of order $n$. Then $C \subseteq V(G \circ H)$ is a weakly convex dominating set of $G \circ H$ if and only if $C=V(G) \cup S$, where $S \subseteq \bigcup_{v \in V(G)} V\left(H^{v}\right)$.

Proof: Let $C$ be a weakly convex dominating set of $G \circ H$. Then $C \cap V(G) \neq$ $\varnothing$. Hence, by Theorem 2.1(a), $C=S_{1} \cup S$, where $S_{1} \subseteq V(G)$ and $S \subseteq$ $\bigcup_{v \in s_{1}} V\left(H^{v}\right)$. If there exists $v \in V(G) \backslash S_{1}$, then $V\left(H^{v}\right) \cap C=\varnothing$. This implies that $C$ does not dominate $V\left(H^{v}\right)$, contrary to our assumption. Therefore, $S_{1}=V(G)$ and $C=V(G) \cup S$.

For the converse, suppose that $C=V(G) \cup S$, where $S \subseteq \bigcup_{v \in V(G)} V\left(H^{v}\right)$. Since $V(G)$ is a weakly convex dominating set of $G$, it follows from Theorem 2.1 that $C$ is a weakly convex dominating set of $G \circ H$.

The next result follows from Theorem 3.1.

Corollary 3.2 Let $G$ be a connected graph of order $m \geq 2$ and $H$ a graph of order $n$. Then $C \subseteq V(G \circ H)$ is a minimum weakly convex dominating set of $G \circ H$ if and only if $C=V(G)$. In particular, $\gamma_{w c o n}(G \circ H)=m$.

\section{Weakly Connected Independent Domination}

The next result characterizes the weakly connected independent dominating sets of $G \circ H$.

Theorem 4.1 Let $G$ be a connected graph and $H$ any graph. Then $C \subseteq$ $V(G \circ H)$ is a weakly connected independent dominating set of $G \circ H$ if and only if $C \cap V(G)$ is a weakly connected independent dominating set of $G$ and $C \cap V\left(v+H^{v}\right)$ is an independent dominating set of $v+H^{v}$ for every $v \in V(G)$.

Proof: Suppose that $C$ is a weakly connected independent dominating set of $G \circ H$. It is easy to see that $C \cap V(G)$ is weakly connected and an independent set of $G$, and $C \cap V\left(v+H^{v}\right)$ is an independent dominating set of $v+H^{v}$ for every $v \in V(G)$. Suppose $C \cap V(G)$ is not a dominating set of $\mathrm{G}$. Then there exist $u \in V(G) \backslash C$ such that $u z \notin E(G)$ for all $z \in C \cap V(G)$. This means that $\left\langle C \cap V\left(u+H^{u}\right)\right\rangle_{w}$ is a component of $\langle C\rangle_{w}$. This implies that $\langle C\rangle_{w}$ is not connected, contrary to the assumption. Thus, $C \cap V(G)$ is a dominating set of $\mathrm{G}$.

Conversely, suppose that $C \cap V(G)$ is a weakly connected independent dominating set of $G$ and $C \cap V\left(v+H^{v}\right)$ is an independent dominating set of 
$v+H^{v}$ for every $v \in V(G)$. Then $C$ is a weakly connected and dominating set of $G \circ H$. Let $x, y \in C$ with $x \neq y$. If $x, y \in C \cap V(G)$, then $x y \notin E(G \circ H)$. Suppose $x \in C \cap V(G)$ and $y \notin C \cap V(G)$. Then $y \in V\left(v+H^{v}\right)$ for some $v \in V(G)$. Since $x \neq v, x y \notin E(G \circ H)$. Suppose $x, y \notin C \cap V(G)$. If $x, y \in$ $V\left(v+H^{v}\right)$ for some $v \in V(G)$, then $x y \notin E(G \circ H)$. Suppose $x \in V\left(u+H^{u}\right)$ and $y \in V\left(v+H^{v}\right)$ for some $u, v \in V(G)$ with $u \neq v$. Clearly, $x y \notin E(G \circ H)$. This shows that $C$ is an independent set of $G \circ H$.

Theorem 4.2 Let $G$ be a connected graph of order $m$ and $H$ any graph with $i(H) \neq 1$. If $C \subseteq V(G \circ H)$ is a minimum weakly connected independent dominating set of $G \circ H$, then $C \cap V(G)$ is a maximum weakly connected independent dominating set of $G$.

Proof: Suppose that $C$ is a minimum weakly connected independent dominating set of $G \circ H$. Let $C_{1}=C \cap V(G)$ and suppose it is not a maximum weakly connected independent dominating set of $G$. Let $M_{1}$ be a maximum weakly connected independent dominating set of $G$. Then $n=\left|M_{1}\right|-\left|C_{1}\right|>0$. For each $v \in V(G) \backslash C_{1}$, let $D^{v}=V\left(H^{v} \cap C\right)$. Then each $D^{v}$ is an independent dominating set of $v+H^{v}$. Define $C_{2}=C \backslash C_{1}=\bigcup\left\{D^{v}: v \in V(G) \backslash C_{1}\right\}$. Let $v_{0} \in V(G) \backslash C_{1}$ such that $\left|D^{v_{0}}\right|=\min \left\{D^{v}: v \in V(G) \backslash C_{1}\right\}$. Let $D \subseteq V(H)$ be such that $\langle D\rangle \cong\left\langle D^{v_{0}}\right\rangle$. For each $u \in V(G) \backslash M_{1}$, let $M^{u} \subseteq V\left(H^{u}\right)$ with $\left\langle M^{u}\right\rangle \cong$ $\langle D\rangle$. Define $M_{2}=\bigcup\left\{M^{u}: u \in V(G) \backslash M_{1}\right\}$. By Theorem 4.1, $C^{\prime}=M_{1} \cup M_{2}$ is a weakly connected independent dominating set of $G \circ H$. Thus,

$$
\left|C^{\prime}\right|=\left|M_{1}\right|+\sum_{u \in V(G) \backslash M_{1}}\left|M^{u}\right|=\left|C_{1}\right|+\left(m-\left|C_{1}\right|\right)|D|+n(1-|D|) .
$$

But $|C|=\left|C_{1}\right|+\sum_{v \in V(G) \backslash C_{1}}\left|D^{v}\right| \geq\left|C_{1}\right|+\left(m-\left|C_{1}\right|\right)|D|$. Since $i(H) \neq 1$, it follows that

$$
|C| \geq\left|C_{1}\right|+\left(m-\left|C_{1}\right|\right)|D|>\left|C_{1}\right|+\left(m-\left|C_{1}\right|\right)|D|+n(1-|D|)=\left|C^{\prime}\right| .
$$

This contrdicts the hypothesis that $C$ is a minimum weakly connected independent dominating set of $G \circ H$. Therefore, $C \cap V(G)$ is a maximum weakly connected independent dominating set of $G$.

Corollary 4.3 Let $G$ be a connected graph of order $m$ and let $H$ be any graph. Then $i_{w}(G \circ H)=\beta_{w}(G)+\left(m-\beta_{w}(G)\right) i(H)$.

Proof: The corollary clearly holds when $i(H)=1$. Suppose $i(H) \neq 1$. Let $C$ be a minimum weakly connnected independent dominating set of $G \circ H$. Let $C_{1}=C \cap V(G)$ and $C_{2}=C \backslash C_{1}$. For each $u \in V(G) \backslash C_{1}$, let $D^{u} \subseteq V\left(H^{u}\right)$ be an independent dominating set of $H^{u}$. Then $C_{2}=\bigcup\left\{D^{u}: u \in V(G) \backslash C_{1}\right\}$. By Theorem 4.2, $C_{1}$ is a maximum weakly connected independent dominating set of $G$. Thus, $\left|C_{1}\right|=\beta_{w}(G)$. Hence, 


$$
i_{w}(G \circ H)=|C|=\left|C_{1}\right|+\sum_{u \in V(G) \backslash C_{1}}\left|D^{u}\right| \geq \beta_{w}(G)+\left(m-\beta_{w}(G)\right) i(H) .
$$

Next, let $C_{1}$ be a maximum weakly connected independent dominating set of $G$ and $D^{\prime}$ be a minimum independent dominating set of $H$. For each $v \in$ $V(G) \backslash C_{1}$, let $D^{v} \subseteq V\left(H^{v}\right)$ be such that $\left\langle D^{v}\right\rangle \cong\left\langle D^{\prime}\right\rangle$. Let $C_{2}=\bigcup\left\{D^{v}: v \in\right.$ $\left.V(G) \backslash C_{1}\right\}$. By Theorem 4.1, $C^{\prime}=C_{1} \cup C_{2}$ is a weakly connected independent dominating set of $G \circ H$. Thus,

$$
i_{w}(G \circ H) \leq\left|C^{\prime}\right|=\left|C_{1}\right|+\sum_{v \in V(G) \backslash C_{1}}\left|D^{v}\right|=\beta_{w}(G)+\left(m-\beta_{w}(G)\right) i(H) .
$$

Therefore, $i_{w}(G \circ H)=\beta_{w}(G)+\left(m-\beta_{w}(G)\right) i(H)$.

\section{References}

[1] J. E. Dunbar, J. W. Grossman, J. H. Hattingh, S. T. Hedetniemi, A. A. McRae, On Weakly-connected Domination in Graphs, Discrete Mathematics, 167(1997), 261-269.

[2] Harary, Frank, Graph Theory, Addison-Wesley Publishing company, Inc., USA, 1969.

[3] T. W. Haynes, S. T. Hedetniemi, and P. J. Slater, Fundamentals of Domination in Graphs, Marcel Dekker, Inc., 1998.

[4] T. N. Janakiraman and P. J. A. Alphonse, Weak Convex Domination in Graphs, International Journal of Engineering Science, Advanced Computing and Bio-Technology, 1(2010), 1-13.

[5] M. Lemanska, Weakly Convex and Convex Domination Numbers, Opuscula Mathematica, 24(2004), 181-188.

Received: July 11, 2013 